\title{
Моделирование транспорта электронов в малопериодных GaAs/AlAs сверхрешетках для терагерцового диапазона частот
}

\author{
() Д.Г. Павельев ${ }^{+}$А.П. Васильев*, В.А. Козлов ${ }^{+\ddagger}$ Ю.И. Кошуринов ${ }^{+}$, Е.С. Оболенская ${ }^{+}$, \\ С.В. Оболенский ${ }^{+}$В.М. Устинов ${ }^{\bullet}$
}

+ Нижегородский государственный университет им. Н.И. Лобачевского, 603950 Нижний Новгород, Россия

* Научно-технологический центр микроэлектроники и субмикронных гетероструктур

Российской академии наук,

194021 Санкт-Петербург, Россия

¥ Институт фризики микроструктур Российской академии наук,

603087 Нижний Новгород, Россия

- Физико-технический институт им. А.Ф. Иоффе Российской академии наук, 194021 Санкт-Петербург, Россия

E-mail: pavelev@rf.unn.ru, obolensk@rf.unn.ru, vasiljev@mail.ioffe.ru, kozlov@ipm.sci-nnov.ru,vmust@beam.ioffe.ru

(Получена 27 апреля 2016 г. Принята к печати 10 мая 2016 г.)

Методом Монте-Карло рассчитан транспорт электронов в сверхрешетках на основе гетероструктур $\mathrm{GaAs} / \mathrm{AlAs}$ с небольшим числом периодов (6 периодов). Эти сверхрешетки используются в диодах, работающих в качестве гармонических смесителей для стабилизации частоты квантовых каскадных лазеров до 5 ТГц. Рассмотрена зонная структура сверхрешеток с различным количеством монослоев AlAs и проведен расчет вольт-амперных характеристик. Проведено сравнение рассчитанных вольт-амперных характеристик сверхрешеток с экспериментом. Теоретически и экспериментально установлена возможность эффективного применения таких сверхрешеток в терагерцовом диапазоне частот.

\section{1. Введение}

В настоящее время происходит интенсивное продвижение когерентной спектроскопии в терагерцовый (ТГц) диапазон частот [1-3]. Преимущество когерентной спектроскопии состоит в том, что в ней используются генераторы эталонных частот микроволнового диапазона. Это позволяет стабилизировать частоту ТГц лазерного излучения с высокой точностью, определяемой точностью эталонного генератора. Такое продвижение оказалось возможным благодаря появлению доступных квантовых каскадных лазеров [4] и созданию гармонических смесителей для ТГц диапазона [5-8]. В неохлаждаемых гармонических смесителях сейчас применяются планарные диоды Шоттки и диоды на основе сверхрешеток $(\mathrm{CP})$. Из сравнения результатов статей $[1,2]$ можно заключить, что достигнутые в когерентной спектроскопии максимальные частоты составляют 3 ТГц для диодов Шоттки и 5.3 ТГц для диодов на основе СР, что позволит с высокой точностью измерить спектральные линии органических молекул.

В данной работе описаны подходы к увеличению предельной частоты диодов на основе сверхрешеток c малой площадью активной области $\left(1-10\right.$ мкм $\left.^{2}\right)$ и исследованы характеристики их омических контактов на основе InGaAs. Использование высоколегированных СР и омических контактов с малым приведенным сопротивлением обусловило возможность применения таких диодов в приборах с рабочими частотами вплоть до нескольких терагерц. В [1] было проведено теоретическое и экспериментальное сравнение параметров 18и 6-периодных сверхрешеток. Было показано, что 6- периодные СР более эффективны и позволяют получать сигнал на гармониках вплоть до 8 ТГц [9]. В настоящей работе путем моделирования методом МонтеКарло более подробно изучен транспорт электронов в 6-периодных СР и выяснена возможность увеличения рабочих частот приборов на сверхрешетках путем оптимизации их параметров и выбора соответствующей конструкции.

\section{2. Объект исследования}

Исследовались планарные диодные структуры, представляющие собой сверхрешетки на основе гетеропары $\mathrm{GaAs} / \mathrm{AlAs}$ с площадью активной области 1-2 мкм². На рис. 1 показана зависимость уровней энергии в таких планарных структурах на основе сверхрешеток от количества монослоев AlAs (от 2 до 6), при фиксированных 18 монослоях GaAs. Данный результат получен путем расчета с использованием модели Кронига-Пенни с учетом поправок, сделанных на основе анализа положения уровней в отдельных квантовых ямах конечной глубины [1]. На вставке к рис. 1 показан профиль потенциала $W$ одного периода СР вдоль кооординаты $z$, перпендикулярной осям СР. Данные рис. 1 показывают, что положение и ширина мини-зоны СР существенно зависят от толщины слоев AlAs, что обусловливает значительные различия в количестве инжектируемых в СР электронов из контактов. Поэтому ток таких СР был существенно различным, что фиксировалось в эксперименте. Эта информация позволила калибровать метод расчета положения дна и потолка мини-зоны в переходных областях СР. 


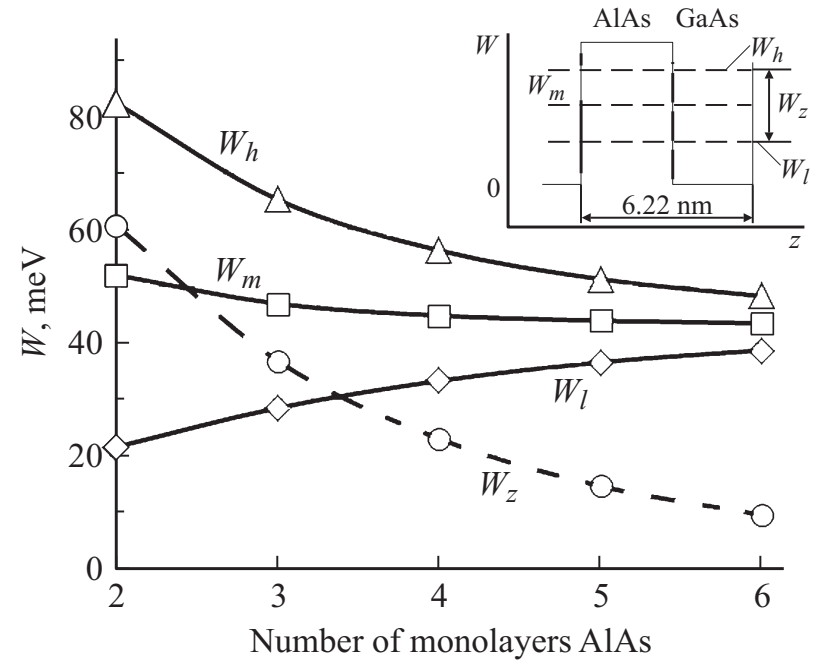

Рис. 1. Результаты расчета положения и ширины мини-зоны СР от количества монослоев AlAs при постоянном количестве монослоев GaAs, равном 18. $W_{h}$ - потолок мини-зоны, $W_{m}-$ середина мини-зоны, $W_{l}$ - дно мини-зоны, $W_{z}=W_{h}-W_{l}-$ ширина мини-зоны. Расчет проведен с использованием модели Кронига-Пенни.

На рис. 2 схематически изображена конструкция короткой диодной структуры на основе 6-периодной СР, показаны зонная диаграмма мини-зоны $W(z)$ с учетом переходных слоев, распределения концентрации доноров и напряженности электрического поля, формируемого в СР при подаче питания. Также приведены описание слоев диодной структуры и ее эквивалентная схема, которая использовалась для расчета сопротивления приконтактных областей и инжектора. Эти сопротивления необходимо учитывать при расчетах величины падения напряжения на рабочей области СР.

Для экспериментальных исследований были изготовлены планарные диодные структуры на основе сверхрешеток $\mathrm{GaAs} / \mathrm{AlAs}$ с малой площадью активной области $\left(1-2\right.$ мкм $\left.^{2}\right)$. Исследуемые структуры выращивались методом молекулярно-лучевой эпитаксии. На полуизолирующей подложке GaAs были последовательно выращены: высоколегированный слой $n^{+}-\mathrm{GaAs}\left(\mathrm{Si}, 6 \cdot 10^{18} \mathrm{~cm}^{-3}\right)$; сверхрешетка $(\mathrm{GaAs})_{18} /(\mathrm{AlAs})_{4}$ с переходными градиентными слоями; высоколегированный слой $n^{+}-\mathrm{GaAs}(\mathrm{Si}$, $\left.6 \cdot 10^{18} \mathrm{~cm}^{-3}\right)$ и слой InGaAs, где мольная доля InAs и концентрация донорной примеси линейно менялись по толщине слоя от 5 до $50 \%$ и от $6 \cdot 10^{18}$ до $10^{19} \mathrm{~cm}^{-3}$ соответственно.

\section{3. Математическая модель}

Для расчетов транспорта электронов совместно использовался одночастичный метод Монте-Карло и квазигидродинамическая модель [10]. Величины падающего напряжения и напряженности поля в сверхрешетке определялись на основе квазигидродинамической модели так, как это описано в [1]. Предполагалось, что электрическое поле однородно в латеральной плоскости, не изменяется во времени и приложено вдоль оси СР. В качестве основных механизмов рассеяния рассматривалось рассеяние на акустических фононах, полярных оптических фононах, атомах ионизованной примеси. Все параметры материала, необходимые для расчета частот рассеяния, выбирались такие же, как и для объемного GaAs. Расчет проводился для комнатной температуры.

При моделировании переноса электронов с использованием метода Монте-Карло предполагалось, что все
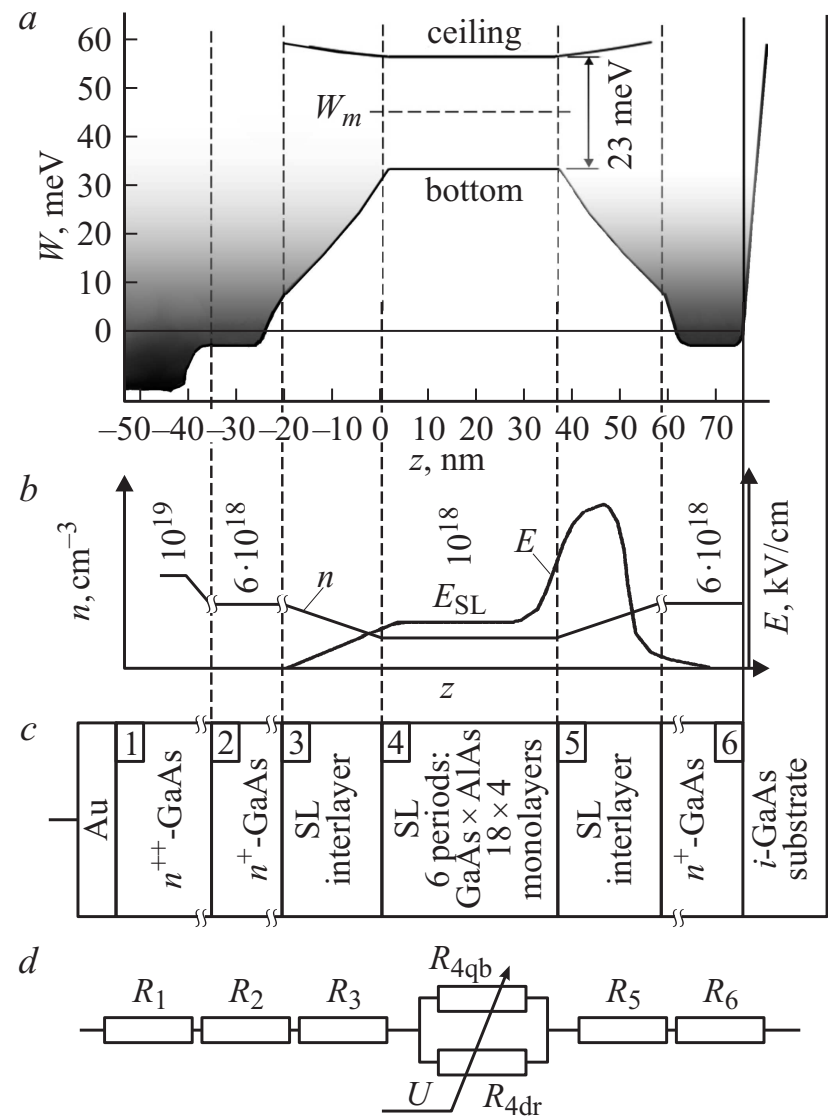

Рис. 2. Конструкция короткой диодной структуры на основе 6-периодной сверхрешетки (SL): $a$ - зонная диаграмма мини-зоны с учетом переходных слоев, градиентной заливкой показано распределение электронов по энергии; $b-$ концентрация электронов $n$ и распределение электрического поля $E$ при внешнем напряжении, $E_{\mathrm{SL}}-$ напряженность поля в сверхрешетке; $c-$ описание слоев $(1-6)$ диодной структуры; $d-$ эквивалентная схема, где нумерация элеметов отражает нумерацию слоев. $W_{m}-$ середина мини-зоны. На краях сверхрешеток сформированы переходные слои, состоящие из чередующихся слоев $\mathrm{AlAs}$ и GaAs с толщинами $1 \times 28$, $2 \times 24$ и $3 \times 21$ монослоев указанных полупроводников. Это позволяло повысить эффективный ввод электронов в сверхрешетку. Сопротивление рабочей области сверхрешетки определяется двумя различными по физической природе потоками электронов - квазибаллистическим и дрейфовым движением носителей заряда, поэтому на эквивалентной схеме указаны два сопротивления $\left(R_{4 \mathrm{qb}}, R_{4 \mathrm{dr}}\right)$ величины которых по-разному зависят от внешнего напряжения $U$. 
электроны расположены в нижней мини-зоне СР и вероятность перехода электрона в рабочей области в более высоко лежащую мини-зону мала. Использовалось обычное соотношение для зависимости энергии электрона от волнового вектора. Особое внимание обращалось на определение доли квазибаллистических электронов и подсчет количества малоугловых рассеяний, a также определялась доля электронов, претерпевших существенное изменение траектории движения за счет рассеяния на оптических фононах. Последнее позволило установить роль квазибаллистического транспорта в коротких сверхрешетках и определить вклад указанных электронов в проводимость СР.

В ходе выполнения расчетов моделировалось движение электронов с учетом их прохождения через сверхрешетку или отражения в область инжектора, т.е. в левый контакт (рис. 2). Рассчитывались зависимости средней скорости, полной и поперечной энергии, а также концентрации электронов от координаты. Расчеты повторялись для каждого варианта положения мини-зоны, обусловленного толщиной слоя AlAs, как это показано на рис. 1. Кроме того, проводился модельный расчет для соотношения слоев GaAs и $\mathrm{AlAs}$, равного $18 \times 4$. Положение мини-зоны варьировалось, и снова проводились расчеты величины электрического тока, которые затем сравнивались с экспериментом с целью калибровки модели. Последнее позволяло точнее определить вклад сопротивлений приконтактных областей, величину падения напряжения на сверхрешетке и напряженность поля в ней при заданном внешнем напряжении.

\section{4. Результаты расчетов и их обсуждение}

В результате моделирования были получены зависимости, приведенные на рис. 3-6. Также были рассчитаны количество одновременно движущихся в СР электронов, доля баллистически прошедших и отраженных электронов, максимальная и средняя длина свободного пробега для разной напряжённости поля внутри СР и различных положений мини-зоны. Результаты расчетов приведены в табл. $1-3$.

Приведенная на рис. 3 зависимость средней скорости электронов $\langle v\rangle$ от координаты $z$ имеет нарастающий характер, что связано с разгоном электронов в правой части сверхрешетки [11] в диапазоне координаты 25-37 нм. Указанные электроны успевают набрать значительную скорость при энергии ниже потолка минизоны, а затем вылетают из СР, не успев отразиться от потолка мини-зоны. По данным зависимостям был рассчитан интервал средних времен пролета квазибаллистическими электронами рабочей области СР. В зависимости от приложенного поля, эти времена пролета попадали в интервал от 80 до 300 фс.

Из рис. 4 видно, что полная средняя энергия электронов в 1.5-2 раза больше, чем поперечная, что свидетельствует о баллистическом характере движения электронов в рабочей области СР. В целом зависимость
Таблица 1. Количество одновременно движущихся электронов для разной напряженности поля и разного положения мини-зоны (модельный расчет)

\begin{tabular}{c|c|r|c|c}
\hline \multirow{2}{*}{$\begin{array}{c}\text { Положение середины } \\
\text { мини-зоны } W_{m}, \text { мэВ }\end{array}$} & \multicolumn{4}{|c}{ Напряженность поля } \\
\cline { 2 - 5 } & 2 кВ/см & 8 кВ/см & 12 кВ/см & 20 кB/см \\
\hline 20 & 1320 & 8310 & 4960 & 10400 \\
30 & 1550 & 43800 & 16200 & 49300 \\
40 & 1780 & 9220 & 12900 & 52600 \\
45 & 1890 & 8650 & 13700 & 35400 \\
50 & 2060 & 8220 & 14400 & 16900
\end{tabular}

Примечание. Соотношение слоев GaAs и AlAs $18 \times 4$.

Таблица 2. Доля баллистических электронов (\%) для разной напряженности поля и положения середины мини-зоны $W_{m}=45$ мэВ

\begin{tabular}{c|c|c|c|c}
\hline \multirow{2}{*}{$\begin{array}{c}\text { Количество рассеяний } \\
\text { на оптических фононах }\end{array}$} & \multicolumn{4}{|c}{ Напряженность поля } \\
\cline { 2 - 5 } & 2 кВ/см & 8 кВ/см & 12 кВ/см & 20 кВ/см \\
\hline 0 & 5 & 3 & 2 & 0 \\
$1-2$ & 22 & 15 & 9 & 6 \\
$3-5$ & 19 & 12 & 10 & 10 \\
$5-10$ & 6 & 7 & 8 & 9 \\
$>10$ & 0 & 1 & 0 & 0 \\
отражение & 48 & 62 & 71 & 75
\end{tabular}

Примечание. Соотношение слоев GaAs и AlAs $18 \times 4$.

Таблица 3. Средняя длина свободного пробега (нм) для разной напряженности поля и разного положения мини-зоны (модельный расчет)

\begin{tabular}{c|l|l|l|l}
\hline \multirow{2}{*}{$\begin{array}{c}\text { Положение середины } \\
\text { мини-зоны } W_{m} \text {, мэВ }\end{array}$} & \multicolumn{4}{|c}{ Напряженность поля } \\
\cline { 2 - 5 } & 2 кB/cм & 8 кB/см & 12 кB/см & 20 кВ/см \\
\hline 20 & 0.03 & 0.14 & 0.98 & 0.23 \\
30 & 0.215 & 0.242 & 0.34 & 0.123 \\
40 & 0.466 & 0.198 & 0.95 & 0.196 \\
45 & 0.24 & 0.165 & 1.84 & 0.275 \\
50 & 0.11 & 0.135 & 1.738 & 0.375
\end{tabular}

Примечание. Соотношение слоев GaAs и AlAs $18 \times 4$.

энергии от координаты медленно спадает, что объясняется частичным отражением инжектированных в рабочую область электронов назад, в исходный (левый) контакт, a также потерей энергии на излучение оптических фононов и взаимодействием с потолком мини-зоны.

Зависимость концентрации электронов $n$ от координаты (рис. 5) рассчитана с учетом данных о величине прошедших и отраженных электронов, приведенных в табл. 1 и 2. С одной стороны, количество одновременно движущихся электронов достаточно для обоснования используемого в работе приближения о движении электронов в постоянном поле. С другой стороны, количество отраженных электронов для напряженностей поля 8-20 кВ/см близко друг к другу, т. е. можно утверждать, 


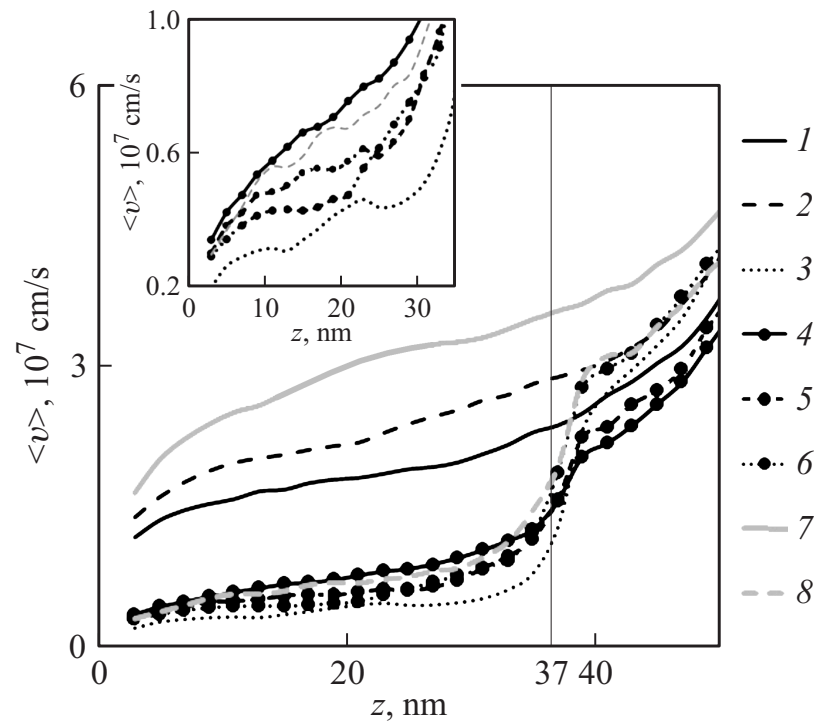

Рис. 3. Результаты расчетов средней скорости в зависимости от координаты. На вставке - начальный участок в увеличенном масштабе. Положение середины мини-зоны $W_{m}$, мэВ/напряженность поля в сверхрешетке $E_{\mathrm{SL}}, \mathrm{\kappa B} / \mathrm{cm}: 1-30 / 8$, $2-20 / 12,3-30 / 20,4-45 / 8,5-45 / 12,6-45 / 20$, $7-30 / 12,8-45 / 12.7,8-$ результаты модельных расчетов, когда рассеяние на примеси отключено.

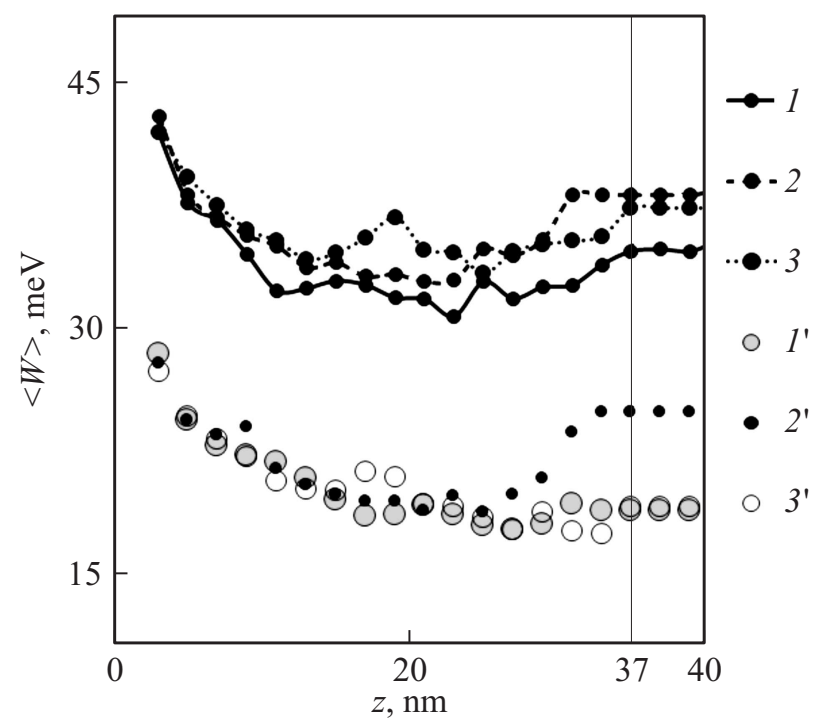

Рис. 4. Результаты расчетов для средней $(1-3)$ и средней поперечной $\left(1^{\prime}-3^{\prime}\right)$ энергий электронов от координаты в СР. Положение середины мини-зоны $W_{m}=45$ мэВ. Напряженность поля в сверхрешетке $E_{\mathrm{SL}}=8\left(1,1^{\prime}\right), 12\left(2,2^{\prime}\right), 20 \mathrm{\kappa B} / \mathrm{cm}\left(3,3^{\prime}\right)$.

что величина инжекции электронов в СР из левого контакта примерно постоянна, т. е. величина электрического тока определяется скоростью носителей заряда чем выше напряженность поля, тем ниже скорость и ток. Иными словами, здесь наблюдается отрицательная дифференциальная проводимость. Кроме того, зависимости скорости и концентрации электронов от координаты носят взаимно обратный характер, а их произведение постоянно, что свидетельствует о постоянстве тока в разных сечениях СР в случае постоянного напряжения питания. Иными словами, в данной модели в стационарном состоянии нет сильного накопления заряда, а плотность тока вдоль СР постоянна.

На рис. 6 приведено сравнение экспериментальной и расчетной вольт-амперных характеристик $I(U)$. Отмече-

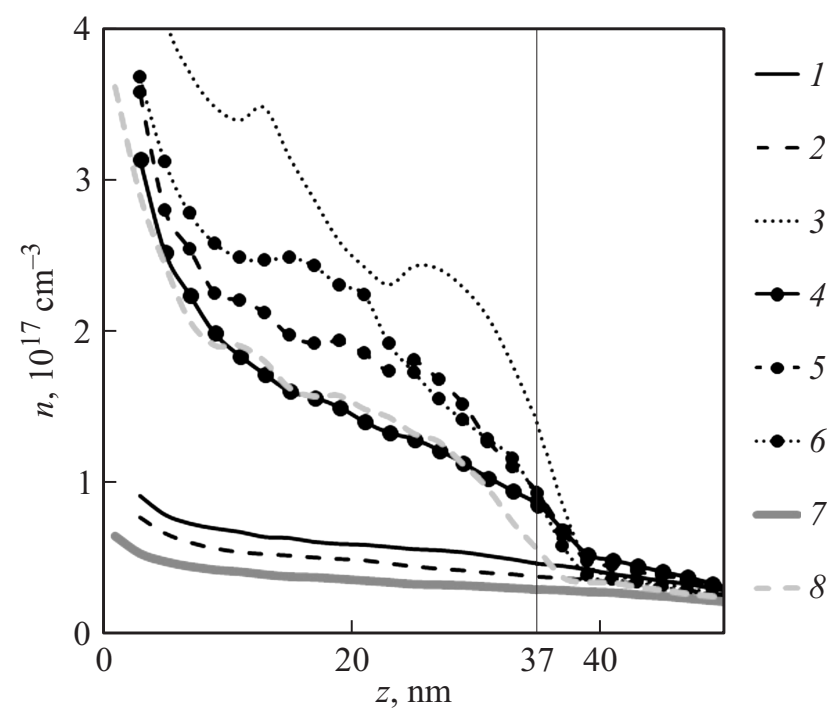

Рис. 5. Результаты расчетов зависимости концентрации электронов от координаты в СР. Положение середины минизоны $W_{m}$, мэВ/напряженность поля в сверхрешетке $E_{\mathrm{SL}}$, кВ/см: $1-30 / 8,2-20 / 12,3-20 / 20,4-45 / 8,5-45 / 12$, $6-45 / 20,7-30 / 12,8-45 / 12.7,8-$ результаты модельных расчетов, когда рассеяние на примеси отключено.

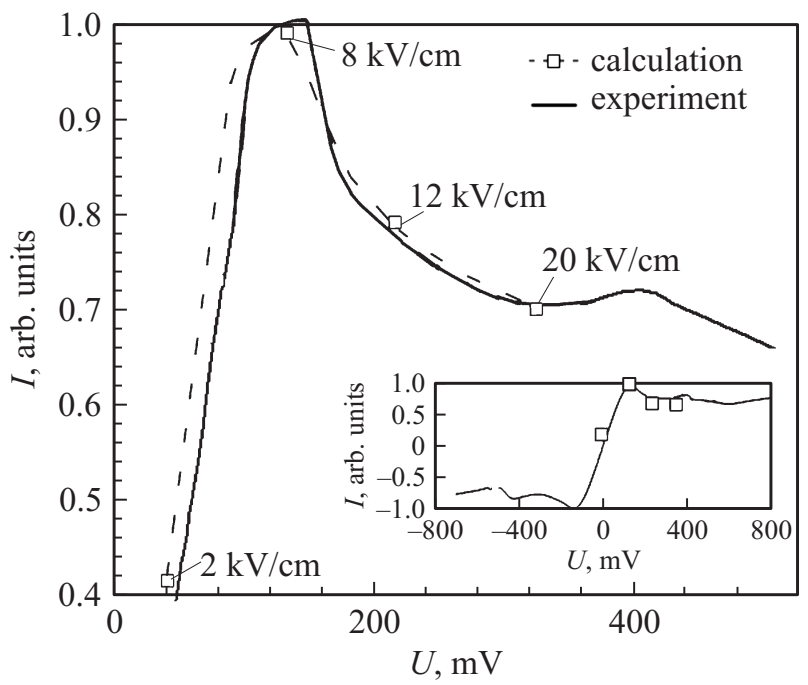

Рис. 6. Экспериментальная и рассчитанная вольт-амперные характеристики $I(U)$ для 6-периодной СР. Соотношение напряженности поля в СР и внешнего напряжения питания $U$ определялось с учетом сопротивлений контактов, указанных на эквивалентной схеме (рис. $2, b$ ), согласно подходу, изложенному в [1]. 
ны расчетные точки, и приведены соответствующие им напряженности электрических полей, реализующихся в CP с учетом падения напряжения на сопротивлениях контактов, согласно эквивалентной схеме, приведенной на рис. 2. В ходе вычислений получено, что соответствие величины тока достигается при скоростях $(1-9) \cdot 10^{6} \mathrm{~cm} / \mathrm{c}$, которые реализуются при положении мини-зоны с $W_{m}=45$ мэВ для $\mathrm{CP}$ с соотношением монослоев GaAs и AlAs, равным $18 \times 4$. Правильность такого положения мини-зоны подтверждает модельный расчет: при смещении мини-зоны скорости электронов существенно увеличиваются из-за изменения энергии инжектируемых электронов во входной области СР. Это приводит к иному значению величины тока. Ни то, ни другое не соответствует экспериментальным данным, a также известным данным о характерных скоростях электронов в сверхрешетках рассматриваемого типа [8].

С целью анализа характера движения электронов в СР был проведен расчет доли баллистических, квазибаллистических и отраженных электронов для разных напряженностей электрического поля и положений мини-зоны в СР (табл. 2). Видно, что с увеличением напряженности поля растет количество отброшенных назад электронов, а доля пролетевших рабочую область баллистических электронов, т.е. электронов, не претерпевающих рассеяние, падает. Важно, что для более длинных СР вероятность квазибаллистического движения падает и будет большую роль играть группировка электронов связанная с рассеянием, схожая по своему принципу с группировкой в диодах Ганна [8]. Другим важным следствием, вытекающим из данных табл. 2 и рис. 3 , является тот факт, что примесное рассеяние, которое здесь является малоугловым, слабо сказывается на скорости электронов в сверхрешетках с малым числом периодов. Это важно для проведения оптимизации конструкции CP, так как паразитное сопротивление СР сказывается на эффективности ее работы. Иными словами, оптимальный уровень легирования следует определять исходя из оптимальной концентрации электронов, а не их скорости.

Как было уже получено ранее [1], длина СР, при которой еще сохраняется возможность группировки квазибаллистических электронов, при их скорости $(2-5) \cdot 10^{6} \mathrm{~cm} / \mathrm{c}$ составляет $30-45 \mathrm{HM}$, что как раз соответствует исследуемой структуре. Таким образом, наличие квазибаллистического характера движения электронов повышает эффективность работы на высоких частотах за счет меньшего времени пролета указанных электронов. По нашим результатам видно, что больше всего таких электронов возникает при напряженности поля $8 \mathrm{\kappa B} / \mathrm{cm}$, что соответствует максимуму тока на вольт-амперной характеристике. Затем небольшое увеличение напряжения на СР приводит к резкому снижению тока из-за уменышения ансамбля пролетающих баллистических электронов за счет отражения электронов потолком мини-зоны, что соответствует полям $12 \mathrm{\kappa B} / \mathrm{cm}$ и в большей степени 20 кВ/см.
Как уже отмечалось, результаты моделирования показывают, что характерное время пролета 6-периодной СР квазибаллистическими электронами составляет 80-300 фс в зависимости от напряженности поля в СР. Это означает, что в процессе полета баллистический электрон может совершать достаточно много осцилляций в переменном терагерцовом поле, что обеспечивает достаточно эффективный обмен энергии между этим полем и током, создаваемым баллистическими электронами. Более того, баллистические электроны в коротких гетероструктурах могут приводить к возникновению терагерцовой динамической отрицательной проводимости. Такая отрицательная проводимость возникает на пролетных частотах, определяемых временем пролета электроном характерной длины изменения профиля потенциала гетероструктуры [12-15].

Полученные результаты обосновывают возможность применения таких СР для преобразования сигналов на частотах до 8-12 ТГц. Предельная частота $f_{p}=1 / 2 \pi R_{s} C$, обусловленная влиянием паразитной емкости $C$ активной области диода и его последовательного сопротивления $R_{s}$, рассчитывалась по методике, предложенной в [8], и была равна 10 ТГц. Дополнительные возможности повышения предельных частот СР связаны с уменьшением омического сопротивления контактов малой площади, а также с оптимизацией переходных слоев гетероструктуры (рис. 2), которые могут выступать в роли инжектора баллистических электронов [11] в рабочую область структуры.

Результаты моделирования и сравнение их с экспериментом показали, что одним из важных аспектов создания терагерцовых диодов на основе СР с малым числом периодов является определение оптимального положения и ширины мини-зоны СР. В силу малой длины сверхрешетки средняя скорость носителей заряда внутри СР зависит от энергии вбрасываемых электронов, что существенно сказывается на их быстродействии. С другой стороны, ширина и положение мини-зоны обусловливают количество вбрасываемых в СР электронов, что в совокупности с их скоростью определяет величину электрического тока и выходную мощность. При больших плотностях тока СР начинает разогреваться, и экспериментально было показано, что при длине, равной 6 периодам, оптимальной является СР с соотношением монослоев GaAs и AlAs, равным $18 \times 4$.

\section{5. Заключение}

Результаты проведенного моделирования и сопоставления с экспериментом показывают, что изложенный в работе подход к анализу физических процессов транспорта электронов методом Монте-Карло позволяет проводить анализ деталей процессов переноса носителей заряда, что важно для проведения оптимизации приборов. Предложенные методы калибровки модели по данным экспериментальных исследований крайне важны для последующих работ, так как позволяют проводить 
не только качественные, но и количественные расчеты. Важным выводом здесь является то, что оптимизация СР должна проводиться комплексно с учетом варьирования инжектора, количества периодов СР, ее уровня легирования, положения и ширины мини-зоны, определяемой количеством монослоев GaAs и AlAs.

Представляется, что уменьшение числа периодов позволит еще больше повысить предельные частоты функционирования СР. Проведение оптимизации таких сверхрешеток требует дальнейшего усложнения модели, связанного с введением специальной взаимосвязи энергии и волнового вектора электронов при переходе к штучному количеству уровней в квантовых ямах, формирующих СР.

Успехи, связанные с использованием коротких сверхрешеток в терагерцовом диапазоне частот, свидетельствуют о том, что баллистический пролет электронами коротких гетероструктур со специально подобранным профилем потенциала (без наложения дополнительного ограничения, связанного с периодичностью) может быть использован для создания методами зонной инженерии таких структур, в которых возможно наблюдение высокочастотных пролетных эффектов, в том числе новых типов отрицательной проводимости.

Работа поддержана грантами РФФИ № 14-02-00581, 15-02-07935 и МОН РФ (соглашение от 27 августа 2013 г. № 02.B.49.21.0003), а также государственным заданием МОН РФ (код проекта 2183).

\section{Список литературы}

[1] Д.Г. Павельев, А.П. Васильев, В.А. Козлов, Ю.И. Кошуринов, Е.С. Оболенская, С.В. Оболенский, В.М. Устинов. Журн. радиоэлектроники, 1, 1 (2016).

[2] A. Danilov, N. Erickson, A. Light, J. Waldman. Optics Lett, 40, 5090 (2015).

[3] A.V. Khudchenko, D.J. Hayton, D.G. Pavelyev, A.M. Baryshev, J.R. Gao, T.-Y. Kao, Q. Hu, J.L. Reno, V.L. Vaks. 39 Int. Conf. IRMMWTHz (Tucson, Arisona, 2014).

[4] Р.Ф. Казаринов, Р.А. Сурис. ФТП, 5, 797 (1971).

[5] D.G. Paveliev, Yu.I. Koschurinov, V.M. Ustinov, A.E. Zhukov, F. Lewen, C. Endres, A.M. Baryshev, P. Khosropanah, Wen Zhang, K.F. Renk, B.I. Stahl, A. Semenov, H.-W. Huebers. 19th Int. Symp. Space Terahertz Technology (Groningen, 2008) p. 319.

[6] C.P. Endres, F. Lewen, T.F. Giesen, S. Schlemmer, D.G. Paveliev, Y.I. Koschurinov, V.M. Ustinov, A.E. Zhukov. Rev. Sci. Instr., 78, 043106 (2007).

[7] В.Л. Вакс, Ю.И. Кошуринов, Д.Г. Павельев, А.Н. Панин. Изв. вузов. Радиофизика, 48 (10-11), 110 (2005).

[8] Д.Г. Павельев, Н.В. Демарина, Ю.И. Кошуринов, А.П. Васильев, Е.С. Семенова, А.Е. Жуков, В.М. Устинов. ФТП, 38, 1141 (2004).

[9] Д.Г. Павельев, Ю.И. Кошуринов, А.С. Иванов, А.Н. Панин, В.Л. Вакс, В.И. Гавриленко, А.В. Антонов, В.М. Устинов, А.Е. Жуков. ФТП, 46, 125 (2012).

[10] S.V. Obolensky, A.V. Murel, N.V. Vostokov, V.I. Shashkin. IEEE Trans. Electron Dev., 58 (8), 2507 (2011).

[11] М.В. Безногов, Р.А. Сурис. ФТП, 47, 493 (2013).
[12] V.A. Kozlov, A.B. Kozyrev, A.V. Samokhvalov. Proc. 11th Int. Symp. Ultrafast Phenomena in Semiconductors (Vilnius, 2001) [Mater. Sci. Forum, 384-385, 139 (2002)].

[13] V.A. Kozlov, A.V. Nikolaev, A.V. Samokhvalov. Semicond. Sci. Technol., 19 (4), S99 (2004).

[14] V.A. Kozlov, A.V. Nikolaev, V.A. Verbus. Proc. 14th Int. Conf. on Nonequilibrium Carrier Dynamics in Semiconductors (Chicago, 2005) [Springer Proc. Phys., 110, 257 (2005)].

[15] В.А. Козлов, А.Б. Козырев. ФТП, 30, 2002 (1996).

Редактор Л.В. Шаронова

\section{Simulation of electron transport in GaAs/AIAs superlattices with a small number of periods for terahertz frequency range}

\author{
D.G. Pavelyev ${ }^{+}$, A.P.Vasilev*, V.A. Kozlov ${ }^{+\ddagger}$, \\ Yu.I. Koschurinov ${ }^{+}$, E.S. Obolensky ${ }^{+}$, \\ S.V. Obolensky ${ }^{+}$, V.M. Ustinov ${ }^{\bullet}$ \\ + Lobachevsky State University of Nizhny Novgorod, \\ 603950 Nizhny Novgorod, Russia \\ * Submicron Heterostructures for Microelectronics, \\ Research \& Engineering Center, \\ Russian Academy of Sciences, \\ 194021 St. Petersburg, Russia \\ $¥$ Institute for Physics of Microstructures, \\ Russian Academy of Sciences, \\ 603087 Nizhny Novgorod, Russia \\ - loffe Institute, \\ 194021 St. Petersburg, Russia
}

Abstract The electron transport in superlattices based on the heterostructures GaAs/AlAs with a small number of periods (6 periods) was calculated by Monte Carlo method. These superlattices are used in terahertz diodes for frequency stabilization of quantum cascade lasers till $5 \mathrm{THz}$. The band structure of superlattices with different number of monolayers was considered. The calculated current-voltage plots of superlattices were compared with experiment. The possibility of the effective usage of these superlattices in the terahertz frequency range was established. 